\title{
UNA INDEPENDENCIA DENTRO DE UN ORDEN CONSTITUCIONAL: MÉXICO, 1821
}

\author{
AN INDEPENDENCE WITHIN A \\ CONSTITUTIONAL ORDER: \\ MEXICO, 1821
}

\author{
Jaime del Arenal Fenochio \\ CEID \\ jarefen@yahoo.com.mx
}

\begin{abstract}
On September 28th, 1821, the Independence of the Mexican Empire was declared. However, there has been disregard of the fact that the birth of Mexico was accomplished within the duration of a modern constitutional order, partially and temporarily governed by the Spanish Constitution of 1812 and the principles in the Plan of Iguala and Treaties of Córdoba, regardedas the first Fundamental Laws of the new political body, until the Mexican Congress issued the first truly mexican constitution. This fact and this legal act were unique in Latin American political history and reaffirmed the constitutionalist vocation of those who conceived them.
\end{abstract}

Keywords: constitution, constitucional order, consummation of Independence, Mexico 1821.

\begin{abstract}
Resumen
El 28 de septiembre de 1821 se declaró la Independencia del Imperio Mexicano. Poco se ha reparado en el hecho de que el nacimiento de México se llevó dentro de la vigencia de un orden constitucional moderno, regido parcial y temporalmente por la Constitución española de 1812 y por los principios establecidos en el Plan de Iguala y los Tratados de Córdoba, considerados como las primeras leyes fundamentales, hasta que el congreso mexicano expidiera la primera constitución propiamente mexicana. Este hecho y este acto jurídico fueron únicos en la historia política latinoamericana y reflejaron una vocación constitucionalista.
\end{abstract}

Palabras clave: constitución, orden constitucional, consumación, independencia, México 1821. 
Cuando, en retrospectiva, se analizan comparativamente los últimos eventos y procesos que llevaron a la definitiva independencia de los distintos países que integrarán Latinoamérica, tanto de los que formaron parte de la Monarquía hispánica como de la Corona lusitana, resalta el caso singular novohispano por ser el único que se condujo sobre el respeto de la vigencia de un orden constitucional moderno, previamente establecido por la Constitución española sancionada en Cádiz el 18 de marzo de 1812 (Tena Ramírez, 1964); si bien, dicho orden hubo de reconocerse en forma parcial y transitoria, en tanto el nuevo Estado independiente se daba a sí mismo su propia constitución, por no ajustarse la española del todo a la realidad política, social y económica de la Nueva España.

No obstante la importancia de este hecho, muy pocos autores -entre historiadores y juristas, mexicanos y extranjeros- lo han observado debido a que la historiografía de la época no lo analizó y, posteriormente, en medio de la polémica que suscitó el establecimiento del Imperio mexicano y los afanes republicanos de una parte de la élite política de aquellos años, nadie quiso reparar en la propuesta de una independencia dentro de un orden constitucional y menos en atribuirle este camino a quien optó por continuar con la forma monárquica constitucional de gobierno, si bien limitándola a una constitución "adaptable al reino". Fue, ciertamente, más fácil, cómodo y útil a la historiografía republicana calificar el hecho de la consumación de la Independencia como un movimiento reaccionario, dirigido a reestablecer el absolutismo político en la persona del emperador Agustín I.

También ha podido influir, en esta consideración, el hecho de que el plan suscrito en Iguala el 24 de febrero de 1821 se haya querido entender a la manera de los planes políticos -muchísimos- que a partir de 1822 surgirán en la República mexicana para postular, de manera violenta, formas y propuestas de gobierno, nuevas constituciones o radicales reformas sociales y económicas, hasta bien entrado el siglo XX (Ulloa y Her- nández Santiago, 1987). Por el contrario, el Plan de Yndependencia (Del Arenal Fenochio, 2002a), proclamado por Agustín de Iturbide (1783 - 1824) el 1 y 2 de marzo en la mencionada población, no contuvo un programa llamado a desconocer el orden constitucional vigente o a levantar las armas en contra de las autoridades legítimas constituidas, ni menos, a convocar a una revuelta popular agresiva y justiciera, dirigida a establecer un orden político diametralmente distinto al vigente, calificado de ilegítimo, como, en su momento, sí lo harán planes tan famosos en la historia mexicana, por ejemplo los planes de Ayutla (1854), Tuxtepec (1876), San Luis (1910), Ayala (1911), Guadalupe (1913) o el de Agua Prieta (1920). No, el Plan de Iguala convocó al establecimiento y formación de un nuevo Estado independiente -un Imperio-, respetando, hasta donde fuera posible y conveniente, la vigencia de la Constitución de 1812, vuelta a proclamar y jurada en la Nueva España a finales de mayo de 1820 por las autoridades civiles, eclesiásticas, militares y por todas las corporaciones, comunidades religiosas, villas, ciudades y pueblos que formaban parte de una extensa América Septentrional, por entonces en franco proceso de desmembración, gracias a las reformas emprendidas en el siglo XVIII por los últimos reyes de la dinastía borbónica.

Sin embargo, el hecho de llamar a una independencia política absoluta respecto de la Corona española y no a una simple autonomía -como han querido sostener sin fundamento algunos historiadores- supuso, sin duda, un distanciamiento e incluso un relevante rompimiento con parte del contenido previsto por la mencionada Constitución. Esta ambigüedad constituye el verdadero trasfondo constitucional del proceso final de la Independencia de México (Martínez Báez, 1971) y, por su importancia política y jurídica evidente, debe ser objeto de nuevas reflexiones por parte de juristas e historiadores del Derecho, de las instituciones y, desde luego, de la política interesada en el "nacimiento de México".

El Plan de Iguala, en efecto, implicó una crítica a la Constitución de Cádiz, pero nun- 
ca un rechazo a la idea o a la necesidad de contar con una constitución moderna, entendida ésta como el documento político fundamental donde se establece el titular de la soberanía, se limita al poder mediante la división de sus funciones y se consagran ciertos derechos del hombre, entre los cuales los primeros siempre fueron la libertad, la igualdad ante la ley, la propiedad y la seguridad, tal y como lo estableció el precedente constitucional norteamericano y el francés en el siglo XVIII (Fioravanti, 2001).

Así lo reconocería, incluso, el líder indígena insurgente don Pedro Asencio Alquisiras (1786-1821) poco antes de morir, en carta dirigida a don Vicente Guerrero (1782-1831) el 14 de mayo de 1821, donde le confirma que en la Constitución española "está mandado se guarde en lo civil" y su intención de querer "llevar hasta el cavo [sic] el cumplimiento del sistema adoptado" (Del Arenal Fenochio, 2021b: 958-959), superando de esta forma el recelo original de Guerrero frente a la Carta gaditana expresado en la misiva a Iturbide del 20 de enero de ese año (Guerrero, 2014: 72-74).

El Plan y, posteriormente, el Tratado de Córdoba, firmado el 24 de agosto del mismo año (Tena Ramírez, 1964), propuso la necesidad de contar con un texto constitucional de este tipo, sólo que consideró que el gaditano no resultaba adecuado para la Nueva España por las siguientes razones: se trataba de un texto que no reconocía la autonomía política y económica que los reinos, audiencias y capitanías americanos exigían cuando menos desde 1808; tampoco contemplaba una igualdad en la representación ante las Cortes de los diputados americanos respecto de los peninsulares, no obstante el mayor número de pobladores de la América española, ni aceptaba como ciudadanos a negros, mulatos y castas con sangre africana; ${ }^{1}$ por si fuera poco, cierto contenido anticlerical, manifestado sobre todo en los decretos de las Cortes de 1820 en materia de reformas eclesiásticas, se entendieron

1 Es en esto en lo que insistiría Guerrero en su carta a Iturbide para justificar su rechazo inicial a la Constitución española. contrarios a la arraigada religiosidad propia de los americanos del Septentrión.

Además, la Constitución española, al proclamarse como una auténtica constitución imperial (regía desde las islas Baleares hasta las Filipinas sin mayor reconocimiento a las singularidades de tantas y tan diversas geografías y poblaciones), no reparaba en la otra constitución, la tradicional o histórica, que se había ido formando a lo largo de 300 años en cada una de esas regiones, siguiendo la tradición castellana que por entonces destacaría el jurista español Francisco Martínez Marina (1754-1833) en su Teoría de las Cortes (1813), y que, según fray Servando Teresa de Mier (1765-1827), se encontraba prevista, en parte y en su origen, en la Recopilación de las leyes de Indias de 1680, como se encargaría de recordar en el libro XIV de su Historia de la revolución de Nueva España (Guerra, 1986; Esquivel Obregón, 1925).

La naturaleza política del Plan de Iguala - "una obra maestra de política y de saber", según Lorenzo de Zavala (1778 -1836)- quizá se entienda mejor si nos atenemos al título completo que su autor (o quienes lo corrigieron) le dio al documento definitivo y oficial del mismo, enviado a Juan Ruiz de Apodaca (1754 - 1835), otrora virrey y, ahora, conforme a lo dispuesto por la propia Constitución, Jefe Político Superior y Capitán General de la Nueva España: Plan o indicaciones para el gobierno que debe instalarse provisionalmente, con el objeto de asegurar nuestra sagrada religión y establecer la independencia del imperio mejicano, y tendrá el título de Junta gubernativa de la América Septentrional, propuesto por el Sr. Coronel D. Agustín de Iturbide al Exmo. Sr. virrey de Nueva España, Conde del Venadito. ${ }^{2}$

Nótese que se trata de un "Plan o indicaciones", es decir, de una serie de señalamientos, instrucciones y principios para formar un gobierno independiente sobre un

2 Así aparece tanto en el ejemplar enviado al "virrey" Apodaca como en la publicación del mismo hecho en el Suplemento 11 de La Abeja Poblana del 2 de marzo de 1821. Un manuscrito se conserva en el Archivo General de la Nación, Operaciones de Guerra, vol. 1013, fs. 456 (356)-457r (357r). Sobre el carácter definitivo y "oficial" de esta versión puede verse Del Arenal Fenochio (2002a). 
imperio nuevo, pero jamás un plan revolucionario que desconociera radicalmente el orden constitucional establecido. Y si bien la vigencia de la Constitución española pareció ser reconocida únicamente para la persecución de los delitos, conforme a lo dispuesto expresamente en el artículo 21 al mencionar que "Ínterin las Cortes [mexicanas] se establecen se procederá en los delitos con total arreglo a la Constitución española" (Del Arenal Fenochio, 2002a: 117), no puede desconocerse que el movimiento de Iguala dio por hecho y formalmente reconoció dos cosas: tanto la vigencia real y formal de la Constitución en la Nueva España como los juramentos hechos a partir de mayo de 1820 (Ferrer, 1993; Ferrer, 1995), juramentos que no se desconocían; tampoco se negó el juramento de fidelidad al monarca, a tenor de lo dispuesto precisamente por el artículo 7 del mismo Plan, disposición que no se ha sabido entender (por ejemplo, Calvillo, 1974) y que dispuso que la Junta Gubernativa prevista gobernara "a nombre de S.M. [Fernando VII] en virtud del juramento de fidelidad que le tiene prestado la Nación" (Del Arenal Fenochio, 2002a: 114). Es decir, ni siquiera hizo falta que se asentara expresamente que la Constitución española seguiría vigente, pues resultaba obvio. ${ }^{3}$

Por otra parte, fue un plan o indicaciones "propuesto... al virrey de Nueva España", es decir, se trató de un plan abierto, público, no un plan sombrío, y si bien hubo de ser maquinado dentro de una secrecía explicable -no tan rígida como cabría suponer-4, estaba llamado no sólo a ser del conocimiento inmediato del "virrey" sino, lo más importante, a reclamar la necesaria e indispensable colaboración de éste y la de todas las autoridades españolas asentadas, tanto en la Península como en la América Septentrional.

3 Si bien no trata del periodo aquí analizado, conviene ver Rojas (2005).

4 Durante el mes de enero hay varios registros de que el plan se estaba fraguando; incluso, Iturbide lo hizo del conocimiento de algunos diputados a Cortes que estaban esperando embarcarse en Veracruz para asistir a las Cortes madrileñas.
Hay que aceptar y entender (y subrayarlo) que el principal propósito que movió a Iturbide para su elaboración fue evitar a toda costa un nuevo estado de guerra civil en la Nueva España, enfrentamiento que amenazaría, otra vez, vidas y haciendas de sus pobladores, sin distinción de su origen, y quizá consumaría la total desmembración de la extensa América Septentrional. ${ }^{5}$ No se quería reactivar la guerra sino sustituirla con la conciliación pacífica de muy diversos intereses. Por ende, se explican tanto la aparente ingenuidad de las cartas que Iturbide dirigió al Conde del Venadito el 24 de febrero, como su furiosa reacción ante la respuesta impolítica y autoritaria de éste (Olagaray, 1924; Del Arenal Fenochio, 2006). Como también se comprenden las misivas dirigidas al comandante de la Nueva Galicia, José de la Cruz (1786-1856), al arzobispo de México y al obispo de Guadalajara, e incluso al rey Fernando y a las mismas Cortes (Olegaray, 1924). Iturbide, sin duda, tenía plena confianza en que aceptarían su plan, ${ }^{6}$ como lo aceptaría posteriormente en Córdoba don Juan O’Donojú (1762 - 1821), dado que respetaba los derechos de los españoles residentes en América, como aseguraban los intereses de la propia Corona española al garantizar como emperador de México a la persona misma del rey Fernando o a alguno de su familia. ${ }^{7}$ Lejos de la ingenuidad, todas estas cartas revelan, por el contrario, indudable sagacidad política, sinceridad y confianza en su proyecto político, además de la certeza de que obraba dentro del orden constitucional jurado y reconocido por todos.

La disputa por los alcances que habría que darle a la Constitución española la iniciaría, sin embargo, Ruiz de Apodaca, al contestar

5 Así lo advierte en sus Memorias escritas desde Liorna (Iturbide, 2001: 42-43).

6 El domingo 25 de febrero, su esposa, Ana Huarte, recibió instrucciones de Iturbide en el sentido de prepararse a recibirlo junto con su padre y toda su familia en México una semana después, advirtiéndole confiadamente "que no entrase en cuidados pues todo estaba ya previsto y allanado" (Fonte en Navarro García, 2014: 185-189).

7 Si bien el Plan previó en última instancia para ceñirse la corona imperial mexicana al archiduque Carlos de la Casa de Austria, el Tratado, por influencia de O'Donojú, la limitó a los miembros de la dinastía borbona. 
las cartas del 24 de febrero el martes 27 de ese mismo mes. Aquel día Iturbide confiaba que éste aceptaría de buen grado el plan que le enviaba en los siguientes términos:

Excelentísimo señor:

Como me persuado firmemente que vuestra excelencia aceptará el proyecto que le propongo hoy de oficio número inmediato anterior, juzgo necesario pasar a sus superiores manos, como lo verifico, un plan de indicaciones y noticias para gobierno de la junta que trata para los casos, tiempos y circunstancias que en el mismo se expresa.

Celebraré que el éxito corresponda a mis sanos deseos e intención recta (Del Arenal Fenochio, 2006: 53, cursivas añadidas).

El 27 de febrero, Ruiz de Apodaca le contestaba calificando su "proyecto de independencia" como "anticonstitucional" y, en consecuencia, esperaba "que vuestra señoría lo separe inmediatamente de sí, y la prueba de esto será seguir su fidelidad al rey y en observar la constitución que hemos jurado". Ese mismo día, el Conde del Venadito previno al teniente coronel Mateo Quilty a no obedecer "las órdenes del señor coronel don Agustín de Iturbide que sean opuestas a los deberes que imponen a usted su empleo y los juramentos que tiene prestados al rey y a la Constitución" (Del Arenal Fenochio, 2006: 54, cursivas añadidas).

La polémica estaba servida. El 3 de marzo, desconcertado y airado a la vez, Iturbide contestó a Ruiz de Apodaca en los siguientes términos:

Con atraso notable ha llegado a mis manos el superior oficio de vuestra excelencia de 27 del próximo pasado, y siento que vuestra excelencia no haya abierto mi carta, porque le escribía de oficio y particularmente, manifestándole el estado peligrosísimo en que se halla el reino y le proponía el remedio para cortar tan graves males. De nada menos adolece mi plan que de sistema anticonstitucional; tengo la ilustración necesaria para conocer los derechos del hombre libre, nada menos quiero que mi engrandecimiento, y el despotismo sólo pueden apreciarlo los mismos déspotas que quieren poner el pie sobre todos los demás (Del Arenal Fenochio, 2006: 55, cursivas añadidas).

En estas dos cartas se exhiben dos lecturas diferentes de la Constitución. Lecturas que, de alguna manera, anticipan y revelan las polémicas en torno a lo que ha significado una constitución moderna y que, por no comprenderse cabalmente desde la perspectiva histórica, han llevado a los juristas modernos, particularmente a los constitucionalistas mexicanos del siglo $X X$ revolucionario, a calificar -como Ruiz de Apodaca mismo- de anticonstitucional al Plan de Iguala. Pero, ¿realmente lo fue? Iturbide se apresuraría a negarlo enfáticamente aduciendo dos elementos para justificar la constitucionalidad de su plan: los derechos del hombre libre y su aversión al despotismo; es decir, la necesidad de imponerle límites al poder del gobernante. Nada menos que lo que más tarde definirá la teoría constitucional como las partes dogmática y orgánica de las constituciones liberales. Esto es, lo propio, lo básico, lo esencial de toda constitución moderna: defensa de los derechos humanos y limitación del poder político. ${ }^{8}$

No cabe duda, en consecuencia, que en el pensamiento y en la decisión de Iturbide, y de sus correligionarios y corresponsales de esos meses de enero y febrero de 1821, no estaba apartarse del todo de la senda constitucional establecida en Cádiz y vigente entonces en la Nueva España, sino que su propósito era acomodar, ajustar, dicha senda a la realidad novohispana, tal y como lo señaló expresamente el artículo 3 del Plan ("Gobierno monárquico templado por una constitución análoga al país", en la versión del Plan de Yndependencia, o "Su Gobierno será Monarquía moderada con arreglo a la Constitución peculiar y adaptable del Reyno", en la versión del "Plan o indicaciones...", Del Arenal Fenochio, 2002a: 114); es decir, a la vigencia relativa de una constitución his-

8 Precisamente esto es lo que, en su trágico momento, desconocieron los regímenes nazifascistas en la primera década del siglo XX (Müller, 2011). 
tórica o tradicional; en todo caso, propia y singular. Pero, por encima de todo, se trataba de proclamar esos derechos del hombre y limitar el poder del monarca, fuera español o mexicano. Y precisamente en esto estriba lo constitucional y moderno de dicho plan, independientemente de no desconocer, ni expresa ni tácitamente (salvo en la cuestión de la independencia absoluta y en los temas que se han visto arriba) la vigencia de la Constitución española ni los juramentos otorgados a ella y al monarca. Era ésta, sin duda, una manera inteligente e institucional, pacífica y sabia, de desatar el nudo sin romperlo: ${ }^{9}$ una forma, por demás, constitucional moderna.

No lo entenderían así Ruiz de Apodaca, los últimos jefes realistas, ni las Cortes españolas, pero por otros intereses; como tampoco lo harían -y también por diferentes razones- el propio Fernando VII y el arzobispo de México, Pedro Fonte (1777 - 1839): unos por aferrarse al poder mediante una lectura exegética o literal de la Constitución y otros por mantener el absolutismo real. Iturbide, por su lado, encaminaría el establecimiento del nuevo Estado -el Imperio mexicano- por una senda constitucional trazada tomando como base y fundamento gran parte del texto de la Constitución española y, encima, lo dispuesto, primero, en el Plan Iguala y, después, en el Tratado de Córdoba, concibiendo a estos tres documentos como "leyes fundamentales" del Imperio naciente, en tanto que el congreso o las cortes mexicanas procedían a elaborar y a aprobar una constitución propiamente mexicana, análoga al país y a su gente, y conforme, en lo posible, a su constitución tradicional.

Quienes, como Ruiz de Apodaca en su tiempo, siguen insistiendo en el carácter anticonstitucional del programa Iguala-Córdoba, se aferran a una lectura de la constitución propia de un siglo posterior, cuando la obra del jurista austriaco Hans Kelsen (2016: 2022), nacido en Praga, se impondrá en la formación intelectual de la inmensa mayoría de

9 Iturbide se jactaría con razón que "seis meses bastaron para desatar el apretado nudo que ligaba a los dos mundos" (Iturbide, 2001: 44). los juristas occidentales, sin tomar en cuenta las lecturas o modos de entender la constitución propios de finales del siglo XVIII y de principios del siglo XIX, precisamente cuando surgió dicho programa. iVaya, ni siquiera como la que haría Ferdinand Lassalle (2016) cuarenta años después en Berlín, en su célebre opúsculo ¿Qué es una constitución?!

La lectura del Plan de Iguala y de la actitud de Iturbide frente a la Constitución gaditana por parte los juristas-historiadores del siglo XX se ha contaminado, en efecto, por la manera contemporánea de entender el concepto "constitución", que habrá de ser sometido a la perspectiva histórica si verdaderamente se quiere comprender lo que significó para los autores y detractores de aquél. 10

En consecuencia, para entender el sentido y los alcances de la constitución moderna en sus orígenes hay que transitar un camino diametralmente distinto al seguido por nuestros constitucionalistas formales de los siglos XX y XXI." Habría que comenzar con la lectura del Diccionario de Autoridades (1726-1739), donde el concepto es definido como "Ordenanza, establecimiento, estatuto, reglas que se hacen y forman para el buen gobierno y dirección de alguna República o Comunidad" (RAE, 2012), que hace ya clara referencia a su naturaleza reglamentaria o normativa, pero sin referirse específicamente a los límites del poder del soberano.

Por su parte, en la Encyclopédie (17511772) ni siquiera aparece la palabra "Constitution" referida a la política o a lo político;

10 Así lo han comenzado hacer los autores de la moderna historia conceptual; para el caso que nos ocupa puede verse la voz "Constitución" del Diccionario político y social del mundo iberoamericano. I. Iberconceptos, dirigido por Fernández Sebastián (2009) particularmente para el caso mexicano la autora Elisa Cárdenas Ayala, pp. 383-391. No he podido leer aún la prometedora obra de Fernández Sebastián, Historia conceptual del Atlántico ibérico. Lenguajes, tiempos, revoluciones, editada recientemente por el Fondo de Cultura Económica de España.

11 Siempre resulta iluminadora la obra de Fioravanti (1996 y 2001). Incluso, la llamada Constitución de Apatzingán, de 1814, había sido reacia a aceptar plenamente el nombre de "constitución" y se conformó con el de Decreto constitucional que, según Estrada Michel y Lujambio Irazábal (2013), no superó en su parte orgánica al Reglamento del Congreso del Anáhuac. 
este sitio le correspondió al concepto de Leyes fundamentales, ubicado dentro del rubro "Libertad política", y definidas como aquellas "que determinan la distribución del poder legislativo, del poder ejecutivo de las cosas que dependen del derecho de gentes y del poder ejecutivo de las que dependen del derecho civil, de manera que estos tres poderes están equilibrados entre sí" (Diderot y d'Alembert, 1986: 119-120). De aquí, la influencia se hizo sentir en obras como el primer texto propiamente de Derecho constitucional impreso en México, precisamente en 1822, y obra de Juan Wenceslao Sánchez de la Barquera (1779-1840), donde los conceptos Leyes fundamentales y Constitución comienzan a alinearse..$^{2}$ Barquera, en efecto, afirma los dos principios "de que deben dimanar las leyes fundamentales de una constitución":

debe en primer lugar hacer una formal declaración de los derechos reservados por los ciudadanos al tiempo de celebrar la asociación política; esto es, fijar los términos de la igualdad, los límites de la libertad, y las obligaciones recíprocas que contraen con el Estado, y las que el Estado contrae con los ciudadanos para su prosperidad y subsistencia, [y] debe en segundo lugar, establecer los límites de los poderes en una distribución justa y racional, y enlazarlos para el equilibrio (Barquera, 1991: 113 y 114).

Por último, esta poco precisa definición del término -y de sus alcances- se continuó todavía hacia 1837, en la edición mexicana del Diccionario razonado de legislación de Joaquín Escriche, a cargo de Juan Rodríguez de San Miguel: "La forma o sistema de gobierno que tiene adoptado cada estado" (Escriche, 1993: 149), sin más.

Se puede seguir con la exploración de las doctrinas y los autores de la época para darnos cuenta de que más allá de cualquier

12 "Es necesario convenir en que la constitución o las leyes fundamentales de un estado, no son en último análisis, mas que la auténtica y solemne expresión de las leyes, condiciones o reglas, con que un pueblo quiere ser gobernado" (Barquera, 1991: 111-114; véanse, además, 61 y 68). cuestión técnica relacionada con la pirámide normativa o la jerarquía formal de las leyes, lo que la constitución moderna (o el orden constitucional moderno) supuso desde un principio fue una forma de limitación del poder político, dividiéndolo y acotándolo mediante un texto público y solemne, y estableciendo en él, como parte esencial de la misma, los derechos del hombre, principiando con los de libertad, igualdad ante la ley, propiedad y seguridad.

Pues esto es lo que se propuso precisamente Iturbide en el Plan de Iguala, si bien la libertad religiosa no fuera aceptada. Y sobre estos presupuestos y bases -enriquecidos en el Tratado de Córdoba suscrito por él y por O’Donojú en la Villa de Córdoba el 24 de agosto- se formó el "programa político de Agustín de Iturbide" (Del Arenal Fenochio, 2002b), se condujo el movimiento Trigarante entre febrero y septiembre de 1821, y se estableció el Imperio mexicano, como un verdadero y auténtico imperio constitucional, donde la Constitución española prolongaría su vigencia incluso hasta el año de 1824 (Del Arenal Fenochio, 2021a). Y si aquél marcó cierta distancia respecto de la Constitución española, no fue -como se ha empeñado en afirmar la historiografía oficial sobre la consumación de la Independencia y sobre Iturbide- para regresar o reestablecer el absolutismo monárquico, sino para instaurar un nuevo orden constitucional, regido en parte y en forma transitoria por aquella Constitución y por el Plan de Iguala y los Tratados de Córdoba, entendidos -repito- como "leyes fundamentales" del Imperio, dándole de esta forma una relativa continuidad a la constitución histórica heredada de la Nueva España, y hasta en tanto el Congreso mexicano diera una constitución escrita al nuevo Estado acorde a su historia, sus necesidades y características; es decir, hasta formar y aprobar una "constitución análoga al país". Gracias a este programa, México nació a la vida constitucional moderna dentro de la vigencia -no total ni definitiva- de una constitución moderna: la española de 1812.

Este propósito quedó consignado física e 
iconográficamente no sólo en la numismática de la época, ampliamente conocida, sino incluso en un retrato del emperador conservado en Filadelfia, Estados Unidos, y al que recientemente se refirió Stephan Salisbury, del periódico The Philadelphia Inquirer, donde publica la entrevista realizada a la conservadora de arte Sarah Mastrangelo en torno a dos retratos, uno de Agustín I y otro de su esposa Ana Huarte, pintados por el pintor Josephus Arias Huarte en 1822, que "esconden" debajo dos retratos, uno de Carlos IV y el otro de la reina María Luisa de Parma (Salisbury, 2021). ${ }^{13}$

Lo verdaderamente interesante, para efectos de mi reflexión, es que la mano izquierda del Emperador se posa sobre un grueso libro con encuadernación negra de lujo en cuyo lomo aparece el título "Constitucion Ameri[ca]na”, revelando el propósito imperial de alejarse del modelo constitucional español para regir a esa América Septentrional, independizada en septiembre de 1821 por una constitución escrita propia. $Y$ si bien el libro representado es muy voluminoso, lo que no corresponde al tamaño de las constituciones modernas ${ }^{14}$, no pude dudarse que su inclusión en el retrato exhibe la intención simbólica de contar con una constitución donde se limitara el poder imperial y se establecieran derechos del hombre; constitución por entonces postergada gracias a la ineficacia y soberbia del primer Congreso constituyente mexicano, e infructuosamente realizada en el Proyecto de Constitución del Imperio Mexicano suscrito por los miembros de la comisión respectiva de la Junta Nacional Instituyente el 4 de marzo de 1823, a unos días de la abdicación al trono de Agustín I, siempre emperador constitucional de México (Del Arenal Fenochio, 2021a).

13 Puede verse un video en https://cutt.ly/smA3IQn 14 Alejandro Mayagoitia, con el conocimiento que lo caracteriza en estos temas, opina que el cuadro sigue la tradicional representación que sobre los libros se llevó a cabo en los retratos novohispanos.

\section{Fuentes consultadas}

Archivo General de la Nación, Operaciones de Guerra, vol. 1013, fs. 456 (356)-457r (357r).

Barquera, Juan Wenceslao (1991), Lecciones de política y derecho público para instrucción del pueblo mexicano, México, UNAM (Ed. facsimilar de la de México, Imprenta de Doña Herculana del Villar y socios, 1822).

Calvillo, Manuel (1974), La consumación de la Independencia y la instauración de la república federal. La república federal mexicana. Gestación y nacimiento, vol. 1. México, Departamento del Distrito Federal.

Del Arenal Fenochio, Jaime (2021a), "Un Imperio constitucional: el inédito Proyecto de Constitución del Imperio Mexicano de la Junta Nacional Instituyente (marzo de 1823)", tesis de doctorado, Facultad de Derecho/Universidad Panamericana, México.

Del Arenal Fenochio, Jaime (2021b), "'Callo y obedezco': la desavenencia final entre Pedro Asencio Alquisiras y Vicente Guerrero a través de diez cartas inéditas", en Historia Mexicana, 71 (2) [282], pp. 949-964.

Del Arenal Fenochio, Jaime (2006), "La vía epistolar de la Independencia”, en Beatriz Rojas (coord.) Mecánica política: para una relectura del siglo XIX mexicano. Antología de correspondencia política, México, Instituto Mora/Universidad de Guadalajara, pp. 29-69.

Del Arenal Fenochio, Jaime (2002a), "Una nueva lectura del Plan de Iguala", en Un modo de ser libres. Independencia y Constitución en México (1816-1822), Zamora, El Colegio de Michoacán, pp. 93-119.

Del Arenal Fenochio, Jaime (2002b), "El significado de la Constitución en el programa político de Agustín de Iturbide (1821-1824)", en Un modo de ser libres. Independencia y Constitución en Méxi- 
co (1816-1822), Zamora, El Colegio de Michoacán, pp. 141-164.

RAE (Real Academia Española) (2012), Diccionario de Autoridades (1726-1739), Diccionario histórico de la lengua española, <https://apps.rae.es/DA.html>, 27 de abril de 2021.

Diderot, Denis y d'Alembert, Jean (1986), Artículos políticos de la "Enciclopedia", Madrid, Tecnos.

Escriche, Joaquín (1993), Diccionario razonado de legislación civil, penal, comercial y forense. Con citas del derecho, notas y adiciones por el licenciado Juan Rodríguez de San Miguel, México, IIJ/ UNAM. (Ed. facsimilar de la de Mégico, oficina de Galván a cargo de Mariano Arévalo, 1837).

Esquivel Obregón, Toribio (1925), La constitución de Nueva España y la primera constitución del México independiente, México, Imp. Manuel León Sánchez.

Estrada Michel, Rafael y Lujambio Irazábal, Alonso (2013), "El cauce alterno: el Reglamento de Morelos", El cauce alterno: el Reglamento del Congreso del Anáhuac y los Sentimientos de la Nación, Chilpancingo, 1813. México, AGN/Inacipe, pp. 9-14.

Fernández Sebastián, Javier (dir.) (2009), Diccionario político y social del mundo iberoamericano. Iberconceptos I, Madrid, Fundación Carolina/Centro de Estudios Constitucionales/Sociedad Estatal de Conmemoraciones Culturales.

Ferrer Muñoz, Manuel (1995), La formación de un Estado nacional en México. El Imperio y la República federal: 1821-1835, México, IIJ/UNAM.

Ferrer Muñoz, Manuel (1993), La Constitución de Cádiz y su aplicación en la Nueva España, México, IIJ/UNAM.

Fioravanti, Maurizio (2001), Constitución. De la Antigüedad a nuestros días, Madrid, Trotta.
Fioravanti, Maurizio (1996), Los derechos fundamentales. Apuntes de historia de las constituciones, Madrid, Trotta.

Fonte, Pedro (2014), "Memorias" en Luis Navarro García, El arzobispo Fonte y la independencia de México, Sevilla, Universidad de Sevilla, pp. 131-269.

Guerra, José (seud.) (1986), Historia de la revolución de Nueva España, antiguamente Anáhuac, t. II, México, Instituto Cultural Helénico/FCE. (Ed. facsimilar de la de Londres, en la Imprenta de Guillermo Glindon, 1813).

Guerrero, Vicente (2014), Primero tuve patria... Recopilación documental, Instituto de Estudios Parlamentarios Eduardo Neri / Cámara de Diputados / El Colegio de Guerrero.

Iturbide, Agustín de (2001), Manifiesto al Mundo o sean apuntes para la historia, México, Fideicomiso Teixidor/Libros del Umbral.

Kelsen, Hans (2016), La garantía jurisdiccional de la Constitución (La justicia constitucional), México, IIJ/UNAM.

Lassalle, Ferdinand (2006), ¿Qué es una constitución?, México, Colofón.

Martínez Báez, Antonio (1971), "El trasfondo constitucional del movimiento de Iguala", Revista de la Universidad de México, 26 (4), diciembre, México, UNAM, pp. 11-19.

Müller, Ingo (2011), Los juristas del horror. La "justicia" de Hitler: el pasado que Alemania no puede dejar atrás, Bogotá, Librería Jurídica Álvaro Nora.

Olagaray, Roberto (1924), Colección de documentos históricos mexicanos, t. II, México, Secretaría de Guerra y Marina / Antigua Librería de Murguía.

Rojas, Beatriz (comp.) (2005). Juras, poderes e instrucciones, México, Instituto Mora. 
Suplemento 11 en La Abeja Poblana del 2 de marzo de 1821.

Salisbury, Stephan (2021), "The eye revealed", The Philade/phia Inquirer, 7 de mayo, Filadelfia, The Philadelphia Inquirer, Arte, <https://cutt.ly/kmA3g$\mathrm{fZ}>, 10$ de mayo de 2021.

Tena Ramírez, Felipe (1964), Leyes fundamentales de México, 1808-1964, México, Porrúa.

Ulloa, Berta y Hernández Santiago, Joel (1987), Planes de la Nación Mexicana, México, Senado de la República / El Colegio de México.

Recibido: 3 de mayo de 2021. Reenviado: 5 de mayo de 2021. Aceptado: 22 de junio de 2021.

\section{Jaime del Arenal Fenochio}

Es Abogado por la Escuela Libre de Derecho; cursó estudios de posgrado en Historia del Derecho Indiano e Historia del Derecho Español en la Universidad Complutense de Madrid. Es maestro en Historia de México por la UNAM y doctor en Derecho por la Universidad Panamericana de México. Entre sus líneas de investigación se encuentran historia del Derecho, historia de las ideas jurídicas, historia de la Iglesia, pensamiento conservador mexicano, historia de la educación e historia del constitucionalismo, así como el proceso de consumación de la independencia de México. Entre sus más recientes publicaciones destaca, como autor: "Una nueva lectura del Tratado de Córdoba”, en José Luis Soberanes Fernández y Ana Carolina Ibarra (coords.), El bicentenario de la consumación de la independencia y la conformación del primer Constituyente mexicano, Ciudad de México, UNAM-Instituto de Investigaciones Históricas, pp. 117-157 (2021). 